\begin{tabular}{|c|c|}
\hline $\begin{array}{l}\text { Chemistry of } \\
\text { Metals and Allo }\end{array}$ & $\begin{array}{l}\text { Chem. Met. Alloys } 8 \text { (2015) 16-21 } \\
\text { Ivan Franko National University of Liv } \\
\text { www.chemetali-journal.org }\end{array}$ \\
\hline
\end{tabular}

\title{
Crystal structure of $R_{3} \mathrm{Mg}_{0.5} D \mathrm{Se}_{7}(R=\mathrm{Ce}, \mathrm{Pr} ; D=\mathrm{Si}, \mathrm{Ge})$
}

\author{
Oksana STROK ${ }^{1}$, Marek DASZKIEWICZ ${ }^{2}$, Lubomir GULAY ${ }^{3}$ \\ ${ }^{1}$ Department of Inorganic and Physical Chemistry, Lesya Ukrainka Eastern European National University, \\ Voli Ave. 13, 43009 Lutsk, Ukraine \\ ${ }^{2}$ Institute of Low Temperature and Structure Research, Polish Academy of Sciences, \\ P.O. Box 1410, 50-950 Wroctaw, Poland \\ ${ }^{3}$ Department of Ecology and Protection of the Environment, Lesya Ukrainka \\ Eastern European National University, Voli Ave. 13, 43009 Lutsk, Ukraine \\ * Corresponding author.Tel.: +380 50 2064898; e-mail: Strok_Oxana@ukr.net
}

Received March 02, 2015; accepted June 24, 2015; available on-line September 1, 2015

The crystal structures of four quaternary $R_{3} \mathrm{Mg}_{0.5} D \mathrm{Se}_{7}$ compounds (structure type $\mathrm{La}_{3} \mathrm{Mn}_{0.5} \mathrm{SiS}_{7}$, space group $P 6_{3}$, Pearson code $h P 23, a=10.669(1) \AA, c=6.0611(8) \AA, R 1=0.0252$ for $\mathrm{Ce}_{3} \mathrm{Mg}_{0.5} \mathrm{SiSe}_{7} ; a=10.531(1) \AA$, $c=6.0420(1) \AA, R 1=0.0275$ for $\operatorname{Pr}_{3} \mathrm{Mg}_{0.5} \mathrm{SiSe}_{7} ; a=10.6579(7) \AA, c=6.0430(7) \AA, R 1=0.0249$ for $\mathrm{Ce}_{3} \mathrm{Mg}_{0.5} \mathrm{GeSe}_{7} ; a=10.615(1) \AA, c=6.0329(7) \AA, R 1=0.0406$ for $\operatorname{Pr}_{3} \mathrm{Mg}_{0.5} \mathrm{GeSe}_{7}$ ) were determined by means of X-ray single-crystal diffraction. The $R$ atoms occupy trigonal prisms capped by two additional atoms. The Mg atoms are located in octahedra and the $D$ atoms in tetrahedra.

\section{Rare-earth compounds / Chalcogenides / Crystal structure / X-ray single-crystal diffraction}

\section{Introduction}

The production of new materials with increasingly complex compositions has become a principle direction in modern science and technology. Among the multicomponent systems, an important place is occupied by complex rare-earth chalcogenides $[1,2]$. The compounds with the general formula $R_{3} M D X_{7}$ (space group $\left.P 6_{3}\right)(R=$ lanthanide element, $M=1$ mono-valent element $(\mathrm{Cu}, \mathrm{Ag})$ or $1 / 2$ of a di-valent element (Mn, Fe, Mg), $D=\mathrm{Si}, \mathrm{Ge}, \mathrm{Sn}$, and $X=\mathrm{S}, \mathrm{Se}$ ) are interesting due to possible applications in the field of nonlinear optics.

The existence of quaternary $R_{3} \mathrm{Mg}_{0.5} \mathrm{Si}(\mathrm{Ge}) \mathrm{S}_{7}$ $\left(R=\mathrm{Y}\right.$, La) compounds (space group $\left.P 6_{3}\right)$ was reported in [3]. Lattice parameters were determined for these compounds. Complete crystal structure determinations of the quaternary rare-earth sulfides $\mathrm{La}_{3} \mathrm{Mg}_{0.5} \mathrm{Si}(\mathrm{Ge}) \mathrm{S}_{7}$ and $R_{3} \mathrm{Mg}_{0.5} \mathrm{GeS}_{7}(R=\mathrm{Y}, \mathrm{Ce}, \mathrm{Pr}$, $\mathrm{Nd}, \mathrm{Sm}, \mathrm{Gd}, \mathrm{Tb}, \mathrm{Dy}, \mathrm{Ho}$, and Er) were reported in [4] and [5], respectively.

In this paper we present our results of the structure refinement of $R_{3} \mathrm{Mg}_{0.5} D \mathrm{Se}_{7}(R=\mathrm{Ce}, \mathrm{Pr} ; D=\mathrm{Si}, \mathrm{Ge})$ on single-crystal X-ray diffraction data.

\section{Experimental}

Samples with the nominal compositions $R_{3} \mathrm{Mg}_{0.5} D \mathrm{Se}_{7}$ ( $R=\mathrm{Ce}, \operatorname{Pr} ; D=\mathrm{Si}, \mathrm{Ge}$ ) were prepared by fusion of the high-purity elemental constituents in evacuated silica ampoules. The purity of the starting materials was better than 99.9 wt.\%. In order to exclude the reaction of magnesium with quartz, the ampoules were lined with graphite. The synthesis was carried out in a tube resistance furnace. The ampoules were gradually heated at a rate of $30^{\circ} \mathrm{C}$ per hour up to a maximal temperature of $1150^{\circ} \mathrm{C}$, and kept at this temperature for $3 \mathrm{~h}$. After that they were slowly cooled $\left(10^{\circ} \mathrm{C}\right.$ per $\mathrm{h}$ ) to $600^{\circ} \mathrm{C}$, and annealed at this temperature for $720 \mathrm{~h}$. Subsequently, the ampoules were quenched in air.

X-ray powder diffraction patterns of the samples were recorded using a DRON-4-13 powder diffractometer $\left(\mathrm{Cu} K \alpha\right.$ radiation, $10^{\circ} \leq 2 \theta \leq 90^{\circ}$, step scan mode with a step size of $0.05^{\circ}$ and a counting time of $5 \mathrm{~s}$ per data point). The similarity of the X-ray powder diffraction patterns of $R_{3} \mathrm{Mg}_{0.5} D \mathrm{Se}_{7}(R=\mathrm{Ce}$, Pr; $D=\mathrm{Si}, \mathrm{Ge}$ ) to those investigated in [3-5] allowed us to conclude that these compounds are isostructural. Small single crystals, with the shape of hexagonal prisms, suitable for crystal structure investigations, were selected from the samples. The compositions of these single crystals were determined by microprobe analysis performed with an EDAX PV9800 microanalyzer.

The X-ray intensities data were collected on a KUMA Diffraction KM-4 four-circle diffractometer equipped with a CCD camera (graphitemonochromatized MoK $\alpha$ radiation, $\lambda=0.71073 \AA$ ). 
The raw data were treated with the CrysAlis Data Reduction program [6], taking into account an absorption correction. The intensities of the reflections were corrected for Lorentz and polarization factors. The crystal structures were solved by Patterson methods and refined by the full-matrix least-squares method using SHELXL-97 [7]. The acentric space group $\mathrm{PG}_{3}$ was checked with the PLATON program [8] and no additional symmetry elements were found.

\section{Results and discussion}

Hexagonal unit cells with similar lattice parameters (Table 1) were observed during testing of the single crystals of $R_{3} \mathrm{Mg}_{0.5} D \mathrm{Se}_{7}(R=\mathrm{Ce}, \operatorname{Pr} ; D=\mathrm{Si}, \mathrm{Ge})$. The extinctions were found to be consistent with the space group $P 6_{3}$, which was used for the crystal structure solution and refinement for all the compounds. One site occupied by $R$, one site by $\mathrm{Mg}$, one site by $\mathrm{Si}(\mathrm{Ge})$ and three sites by Se were determined. The positions of the $R, \mathrm{Si}(\mathrm{Ge})$ and $\mathrm{Se}$ atoms are fully occupied. The site occupancy factors for the $\mathrm{Mg}$ atoms refined to values close to 0.50 for all the investigated compounds. In the final cycles the occupancy factors of the $\mathrm{Mg}$ sites were fixed to 0.50 to satisfy charge balance requirements. The crystallographic data and refinement information for $R_{3} \mathrm{Mg}_{0.5} D \mathrm{Se}_{7}(R=\mathrm{Ce}, \mathrm{Pr}$; $D=\mathrm{Si}, \mathrm{Ge}$ ) are summarized in Table 1 , whereas the atomic coordinates and the atomic displacement factors are given in Table 2. The results of the crystal structure refinement for $R_{3} \mathrm{Mg}_{0.5} D \mathrm{Se}_{7}(R=\mathrm{Ce}, \mathrm{Pr}$; $D=\mathrm{Si}, \mathrm{Ge})$ are in good accordance with the structure type $\mathrm{La}_{3} \mathrm{Mn}_{0.5} \mathrm{SiS}_{7}[9]$.

Table 1 Crystallographic data and experimental details for $R_{3} \mathrm{Mg}_{0.5} D \mathrm{Se}_{7}(R=\mathrm{Ce}, \operatorname{Pr} ; D=\mathrm{Si}, \mathrm{Ge})$.

\begin{tabular}{|c|c|c|c|c|}
\hline Empirical formula & $\mathrm{Ce}_{3} \mathrm{Mg}_{0.5} \mathrm{SiSe}_{7}$ & $\mathrm{Pr}_{3} \mathrm{Mg}_{0.5} \mathrm{SiSe}_{7}$ & $\mathrm{Ce}_{3} \mathrm{Mg}_{0.5} \mathrm{GeSe}_{7}$ & $\mathrm{Pr}_{3} \mathrm{Mg}_{0.5} \mathrm{GeSe}_{7}$ \\
\hline Space group & $P 6_{3}$ (No. 173) & $P 6_{3}$ (No. 173) & $P 6_{3}$ (No. 173) & $P 6_{3}$ (No. 173) \\
\hline Unit cell dimensions: & & & & \\
\hline$a(\AA)$ & $10.669(1)$ & $10.531(1)$ & $10.6579(7)$ & $10.615(1)$ \\
\hline$c(\AA)$ & $6.0611(8)$ & $6.0420(8)$ & $6.0430(7)$ & $6.0329(7)$ \\
\hline$V\left(\AA^{3}\right)$ & $599.15(8)$ & $580.3(1)$ & $594.4(8)$ & $588.7(1)$ \\
\hline $\begin{array}{l}\text { Number of formula units } \\
\text { per unit cell } Z\end{array}$ & 2 & 2 & 2 & 2 \\
\hline Calculated density $\left(\mathrm{g} \mathrm{cm}^{-3}\right)$ & 5.697 & 5.812 & 5.910 & 5.980 \\
\hline $\begin{array}{l}\text { Absorption coefficient } \\
\left(\mathrm{mm}^{-1}\right)\end{array}$ & 32.999 & 34.414 & 35.169 & 36.323 \\
\hline$F(000)$ & 864 & 870 & 900 & 906 \\
\hline Crystal color & dark red & dark red & dark red & dark red \\
\hline Crystal size $\left(\mathrm{mm}^{3}\right)$ & $0.06 \times 0.04 \times 0.04$ & $0.08 \times 0.06 \times 0.05$ & $0.06 \times 0.04 \times 0.03$ & $0.10 \times 0.06 \times 0.05$ \\
\hline$\theta$ range for data collection & $3.82-27.48$ & $3.87-27.42$ & $4.03-28.67$ & $4.04-35.63$ \\
\hline Index ranges & $-13 \leq h \leq 13$ & $-13 \leq h \leq 13$ & $-14 \leq h \leq 14$ & $-13 \leq h \leq 15$ \\
\hline & $-13 \leq k \leq 13$ & $-13 \leq k \leq 13$ & $-14 \leq k \leq 14$ & $-17 \leq k \leq 14$ \\
\hline & $-7 \leq l \leq 7$ & $-7 \leq l \leq 7$ & $-8 \leq l \leq 8$ & $-8 \leq l \leq 9$ \\
\hline Reflections collected & 8361 & 7383 & 9341 & 8989 \\
\hline Independent reflections & $\begin{array}{l}903[R \text { (int. })= \\
0.0524]\end{array}$ & $\begin{array}{l}886[R(\text { int. })= \\
0.0522]\end{array}$ & $\begin{array}{l}1026[R(\text { int. })= \\
0.0725]\end{array}$ & $\begin{array}{l}1677[R \text { (int. })= \\
0.0541]\end{array}$ \\
\hline Refinement method & $\begin{array}{l}\text { Full-matrix least- } \\
\text { squares on } F^{2}\end{array}$ & $\begin{array}{l}\text { Full-matrix least- } \\
\text { squares on } F^{2}\end{array}$ & $\begin{array}{l}\text { Full-matrix least- } \\
\text { squares on } F^{2}\end{array}$ & $\begin{array}{l}\text { Full-matrix least- } \\
\text { squares on } F^{2}\end{array}$ \\
\hline $\begin{array}{l}\text { Absolute structure } \\
\text { parameter }\end{array}$ & $-0.03(3)$ & $0.05(3)$ & $0.00(3)$ & $0.03(3)$ \\
\hline Data/restraints/parameters & $903 / 1 / 37$ & $886 / 1 / 37$ & $1026 / 1 / 38$ & $1677 / 1 / 38$ \\
\hline Goodness-of-fit on $F^{2}$ & 0.995 & 0.984 & 1.001 & 0.870 \\
\hline Final $R$ indices $[I>2 \sigma(I)]$ & $\begin{array}{l}R 1=0.0227 \\
w R 2=0.0369\end{array}$ & $\begin{array}{l}R 1=0.0237 \\
w R 2=0.0399\end{array}$ & $\begin{array}{l}R 1=0.0224 \\
w R 2=0.0425\end{array}$ & $\begin{array}{l}R 1=0.0288 \\
w R 2=0.0423\end{array}$ \\
\hline$R$ (all data) & $\begin{array}{l}R 1=0.0252 \\
w R 2=0.0373\end{array}$ & $\begin{array}{l}R 1=0.0275 \\
w R 2=0.0407\end{array}$ & $\begin{array}{l}R 1=0.0249 \\
w R 2=0.0431\end{array}$ & $\begin{array}{l}R 1=0.0406 \\
w R 2=0.0438\end{array}$ \\
\hline Extinction coefficient & - & & $0.0101(3)$ & $0.0037(1)$ \\
\hline $\begin{array}{l}\text { Largest diff. peak and hole } \\
\left(\mathrm{e} \AA^{-3}\right)\end{array}$ & 0.870 and -1.077 & 1.698 and -1.229 & 1.900 and -1.302 & 1.777 and -2.989 \\
\hline
\end{tabular}


Table 2 Atomic coordinates and thermal displacement factors $\left(\AA^{2}\right)$ for $R_{3} \operatorname{Mg}_{0.5} D \operatorname{Se}_{7}(R=\mathrm{Ce}, \operatorname{Pr} ; D=\mathrm{Si}, \mathrm{Ge})$.

\begin{tabular}{|c|c|c|c|c|c|c|}
\hline Atom & Wyckoff & $x / a$ & $y / b$ & $z / c$ & Occupancy & $U_{\text {eq. }}{ }^{\text {a }}$ \\
\hline \multicolumn{7}{|c|}{$\mathrm{Ce}_{3} \mathrm{Mg}_{0.5} \mathrm{SiSe}_{7}$} \\
\hline $\mathrm{Ce}$ & $6 c$ & $0.12660(4)$ & $0.35753(4)$ & $0.0138(1)$ & 1 & $0.00913(9)$ \\
\hline $\mathrm{Mg}$ & $2 a$ & 0 & 0 & $0.277(3)$ & 0.50 & $0.012(1)$ \\
\hline $\mathrm{Si}$ & $2 b$ & $1 / 3$ & $2 / 3$ & $0.4273(6)$ & 1 & $0.0096(8)$ \\
\hline $\mathrm{Se} 1$ & $2 b$ & $1 / 3$ & $2 / 3$ & $0.7995(2)$ & 1 & $0.0112(3)$ \\
\hline $\mathrm{Se} 2$ & $6 c$ & $0.24899(7)$ & $0.16135(7)$ & $0.0280(1)$ & 1 & $0.0105(1)$ \\
\hline $\mathrm{Se} 3$ & $6 c$ & $0.10777(7)$ & $0.58604(7)$ & $0,2820(1)$ & 1 & $0.00948(1)$ \\
\hline \multicolumn{7}{|c|}{$\mathrm{Pr}_{3} \mathrm{Mg}_{0.5} \mathrm{SiSe}_{7}$} \\
\hline $\operatorname{Pr}$ & $6 c$ & $0.12936(4)$ & $0.35702(4)$ & $0.0126(1)$ & 1 & $0.0177(1)$ \\
\hline $\mathrm{Mg}$ & $2 a$ & 0 & 0 & $0.285(3)$ & 0.50 & $0.019(1)$ \\
\hline $\mathrm{Si}$ & $2 b$ & $1 / 3$ & $2 / 3$ & $0.4271(5)$ & 1 & $0.0098(8)$ \\
\hline $\mathrm{Se} 1$ & $2 b$ & $1 / 3$ & $2 / 3$ & $0.7987(2)$ & 1 & $0.0141(3)$ \\
\hline $\mathrm{Se} 2$ & $6 c$ & $0.25084(9)$ & $0.16022(8)$ & $0.0332(1)$ & 1 & $0.0204(1)$ \\
\hline $\mathrm{Se} 3$ & $6 c$ & $0.10534(7)$ & $0.58333(8)$ & $0.2814(1)$ & 1 & $0.0125(1)$ \\
\hline \multicolumn{7}{|c|}{$\mathrm{Ce}_{3} \mathrm{Mg}_{0.5} \mathrm{GeSe}_{7}$} \\
\hline $\mathrm{Ce}$ & $6 c$ & $0.12943(4)$ & $0.35737(3)$ & $0.01435(9)$ & 1 & $0.0086(1)$ \\
\hline $\mathrm{Mg}$ & $2 a$ & 0 & 0 & $0.282(2)$ & 0.50 & $0.005(1)$ \\
\hline $\mathrm{Ge}$ & $2 b$ & $1 / 3$ & $2 / 3$ & $0.4297(2)$ & 1 & $0.0083(3)$ \\
\hline $\mathrm{Se} 1$ & $2 b$ & $1 / 3$ & $2 / 3$ & $0.8114(1)$ & 1 & $0.0101(3)$ \\
\hline $\mathrm{Se} 2$ & $6 c$ & $0.25035(7)$ & $0.15961(7)$ & $0.0304(1)$ & 1 & $0.0100(1)$ \\
\hline $\mathrm{Se} 3$ & $6 c$ & $0.10040(7)$ & $0.58182(7)$ & $0.2762(1)$ & 1 & $0.0084(1)$ \\
\hline \multicolumn{7}{|c|}{$\mathrm{Pr}_{3} \mathrm{Mg}_{0.5} \mathrm{GeSe}_{7}$} \\
\hline $\operatorname{Pr}$ & $6 c$ & $0.13014(3)$ & $0.35744(3)$ & $0.01420(7)$ & 1 & $0.00932(7)$ \\
\hline $\mathrm{Mg}$ & $2 a$ & 0 & 0 & $0.280(1)$ & 0.50 & $0.003(1)$ \\
\hline $\mathrm{Ge}$ & $2 b$ & $1 / 3$ & $2 / 3$ & $0.4293(1)$ & 1 & $0.0088(2)$ \\
\hline $\mathrm{Se} 1$ & $2 b$ & $1 / 3$ & $2 / 3$ & $0.8119(1)$ & 1 & $0.0101(2)$ \\
\hline $\mathrm{Se} 2$ & $6 c$ & $0.25153(6)$ & $0.15964(5)$ & $0.0319(1)$ & 1 & $0.0106(1)$ \\
\hline $\mathrm{Se} 3$ & $6 c$ & $0.09919(6)$ & $0.58105(6)$ & $0.2744(1)$ & 1 & $0.0092(1)$ \\
\hline Atom & $U_{11}$ & $U_{22}$ & $U_{33}$ & $U_{23}$ & $U_{13}$ & $U_{12}$ \\
\hline \multicolumn{7}{|c|}{$\mathrm{Ce}_{3} \mathrm{Mg}_{0.5} \mathrm{SiSe}_{7}$} \\
\hline $\mathrm{Ce}$ & $0.0098(1)$ & $0.0079(1)$ & $0.0097(1)$ & $-0.0008(3)$ & $-0.0007(3)$ & $0.0044(1)$ \\
\hline $\mathrm{Mg}$ & $0.011(2)$ & $0.011(2)$ & $0.015(4)$ & 0 & 0 & $0.005(1)$ \\
\hline $\mathrm{Si}^{\circ}$ & $0.009(1)$ & $0.009(1)$ & $0.008(1)$ & 0 & 0 & $0.0050(6)$ \\
\hline $\mathrm{Se} 1$ & $0.0130(4)$ & $0,0130(4)$ & $0.0074(7)$ & 0 & 0 & $0.0065(2)$ \\
\hline $\mathrm{Se} 2$ & $0.0111(3)$ & $0.0096(3)$ & $0.0122(4)$ & $-0.0015(4)$ & $-0.0004(5)$ & $0.0062(3)$ \\
\hline $\mathrm{Se} 3$ & $0,0087(4)$ & $0.0097(4)$ & $0.0106(4)$ & $-0.0012(4)$ & $-0.0004(3)$ & $0.0051(3)$ \\
\hline \multicolumn{7}{|c|}{$\mathrm{Pr}_{3} \mathrm{Mg}_{0.5} \mathrm{SiSe}_{7}$} \\
\hline $\mathrm{Pr}$ & $0.0141(2)$ & $0.0179(2)$ & $0.0237(2)$ & $-0.0019(3)$ & $-0.0041(3)$ & $0.0097(1)$ \\
\hline $\mathrm{Mg}$ & $0.015(2)$ & $0.015(2)$ & $0.026(5)$ & 0 & 0 & $0.007(1)$ \\
\hline $\mathrm{Si}$ & $0.012(1)$ & $0.012(1)$ & $0.005(1)$ & 0 & 0 & $0.0060(6)$ \\
\hline $\mathrm{Se} 1$ & $0.0179(4)$ & $0.0179(4)$ & $0.0065(6)$ & 0 & 0 & $0.0090(2)$ \\
\hline $\mathrm{Se} 2$ & $0.0292(4)$ & $0.0199(4)$ & $0.0213(4)$ & $-0.0080(5)$ & $-0.0082(5)$ & $0.0191(3)$ \\
\hline $\mathrm{Se} 3$ & $0.0111(4)$ & $0.0173(4)$ & $0.0105(3)$ & $-0.0003(4)$ & $-0.0001(3)$ & $0.0082(3)$ \\
\hline \multicolumn{7}{|c|}{$\mathrm{Ce}_{3} \mathrm{Mg}_{0.5} \mathrm{GeSe}_{7}$} \\
\hline $\mathrm{Ce}$ & $0.0094(1)$ & $0.0074(1)$ & $0.0091(1)$ & $-0.0008(2)$ & $-0.0008(2)$ & $0.0042(1)$ \\
\hline $\mathrm{Mg}$ & $0.003(1)$ & $0.003(1)$ & $0.010(4)$ & 0 & 0 & $0.0018(9)$ \\
\hline Ge & $0.0093(4)$ & $0.0093(4)$ & $0.0062(5)$ & 0 & 0 & $0.0047(2)$ \\
\hline $\mathrm{Se} 1$ & $0.0114(4)$ & $0.0114(4)$ & $0.0075(6)$ & 0 & 0 & $0.0057(1)$ \\
\hline $\mathrm{Se} 2$ & $0.0113(3)$ & $0.0088(3)$ & $0.0121(3)$ & $-0.0012(3)$ & $-0.0006(4)$ & $0.0065(3)$ \\
\hline $\mathrm{Se} 3$ & $0.0083(3)$ & $0.0089(3)$ & $0.0089(3)$ & $-0.0012(3)$ & $-0.0002(3)$ & $0.0048(3)$ \\
\hline \multicolumn{7}{|c|}{$\mathrm{Pr}_{3} \mathrm{Mg}_{0.5} \mathrm{GeSe}_{7}$} \\
\hline $\operatorname{Pr}$ & $0.0096(1)$ & $0.0078(1)$ & $0.0105(1)$ & $-0.0010(1)$ & $-0.0009(1)$ & $0.0043(1)$ \\
\hline $\mathrm{Mg}$ & $0.001(1)$ & $0.001(1)$ & $0.009(3)$ & 0 & 0 & $0.0006(7)$ \\
\hline $\mathrm{Ge}$ & $0.0099(3)$ & $0.0099(3)$ & $0.0066(5)$ & 0 & 0 & $0.0049(1)$ \\
\hline $\mathrm{Se} 1$ & $0.0115(3)$ & $0.0115(3)$ & $0.0073(6)$ & 0 & 0 & $0.0057(1)$ \\
\hline $\mathrm{Se} 2$ & $0.0116(2)$ & $0.0096(2)$ & $0.0127(3)$ & $-0.0016(3)$ & $-0.0004(3)$ & $0.0067(2)$ \\
\hline $\mathrm{Se} 3$ & $0.0092(3)$ & $0.0097(3)$ & $0.0099(3)$ & $-0.0011(2)$ & $0.0002(2)$ & $0.0054(2)$ \\
\hline
\end{tabular}

${ }^{\text {a }} U_{\text {eq. }}$ is defined as one third of the trace of the orthogonalized $U_{i j}$ tensor. The anisotropic temperature factor exponent takes the form: $-2 \pi^{2}\left[h^{2} a *{ }^{2} U_{11}+\ldots+2 h k a * b * U_{12}\right]$. 
Relevant interatomic distances and the coordination numbers of the $R, \mathrm{Mg}$, and $\mathrm{Si}(\mathrm{Ge})$ atoms are listed in Table 3. All the distances are close to the sums of the respective ionic radii [10].

The unit cell and the coordination polyhedra of the $R, \mathrm{Mg}, D, \mathrm{Se} 1, \mathrm{Se} 2$, and $\mathrm{Se} 3$ atoms in the structure of the $R_{3} \mathrm{Mg}_{0.5} D \mathrm{Se}_{7} \quad(R=\mathrm{Ce}, \mathrm{Pr}$;
$D=\mathrm{Si}, \mathrm{Ge})$ compounds are shown in Fig. 1. The $R$ atoms occupy trigonal prisms capped by two additional atoms. The $\mathrm{Mg}$ atoms are located in octahedra and the $D$ atoms in tetrahedra. Every Se1 and Se3 atom is surrounded by a tetrahedron. The $\mathrm{Se} 2$ atoms are surrounded by tetragonal bi-pyramids.

Table 3 Interatomic distances $(\delta)$ and coordination numbers (C.N.) of the $R, \mathrm{Mg}$ and $D$ atoms in $R_{3} \mathrm{Mg}_{0.5} \mathrm{Se}_{7}$ $(R=\mathrm{Ce}, \operatorname{Pr} ; D=\mathrm{Si}, \mathrm{Ge})$.

\begin{tabular}{|c|c|c|c|c|c|c|}
\hline \multirow{2}{*}{\multicolumn{2}{|c|}{ Atoms }} & \multicolumn{4}{|c|}{$\delta(\AA)$} & \multirow{2}{*}{ C.N. } \\
\hline & & $\mathrm{Ce}_{3} \mathrm{Mg}_{0.5} \mathrm{SiSe}_{7}$ & $\mathrm{Pr}_{3} \mathrm{Mg}_{0.5} \mathrm{SiSe}_{7}$ & $\mathrm{Ce}_{3} \mathrm{Mg}_{0.5} \mathrm{GeSe}_{7}$ & $\mathrm{Pr}_{3} \mathrm{Mg}_{0.5} \mathrm{GeSe}_{7}$ & \\
\hline$R$ & $\begin{array}{l}-1 \mathrm{Se} 2 \\
-1 \mathrm{Se} 2 \\
-1 \mathrm{Se} 3 \\
-1 \mathrm{Se} 2 \\
-1 \mathrm{Se} 3 \\
-1 \mathrm{Se} 3 \\
-1 \mathrm{Se} 1 \\
-1 \mathrm{Se} 2\end{array}$ & $\begin{array}{l}2.9710(8) \\
2.9820(8) \\
3.0095(8) \\
3.082(1) \\
3.1114(9) \\
3.1703(8) \\
3.1809(8) \\
3.244(1)\end{array}$ & $\begin{array}{l}2.9326(8) \\
2.9379(8) \\
2.9973(9) \\
3.058(1) \\
3.0909(9) \\
3.1638(8) \\
3.1489(8) \\
3.294(1)\end{array}$ & $\begin{array}{l}2.9699(7) \\
2.9714(7) \\
3.0105(8) \\
3.090(1) \\
3.1048(8) \\
3.1746(8) \\
3.1510(6) \\
3.275(1)\end{array}$ & $\begin{array}{l}2.9580(6) \\
2.9643(6) \\
2.9980(7) \\
3.0721(9) \\
3.0895(7) \\
3.1677(7) \\
3.1364(5) \\
3.2754(9)\end{array}$ & 8 \\
\hline $\mathrm{Mg}$ & $\begin{array}{l}-3 \mathrm{Se} 2 \\
-3 \mathrm{Se} 2\end{array}$ & $\begin{array}{l}2.77(1) \\
2.77(1)\end{array}$ & $\begin{array}{l}2.761(9) \\
2.771(9)\end{array}$ & $\begin{array}{l}2.780(8) \\
2.791(8)\end{array}$ & $\begin{array}{l}2.778(6) \\
2.790(6)\end{array}$ & 6 \\
\hline$D$ & $\begin{array}{l}-1 \mathrm{Se} 1 \\
-3 \mathrm{Se} 3\end{array}$ & $\begin{array}{l}2.230(4) \\
2.284(1)\end{array}$ & $\begin{array}{l}2.245(4) \\
2.281(1)\end{array}$ & $\begin{array}{l}2.306(1) \\
2.3657(9)\end{array}$ & $\begin{array}{l}2.308(1) \\
2.3703(7)\end{array}$ & 4 \\
\hline
\end{tabular}

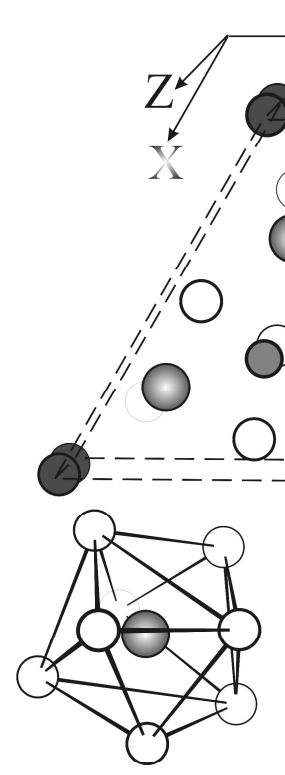

$\mathrm{R}$

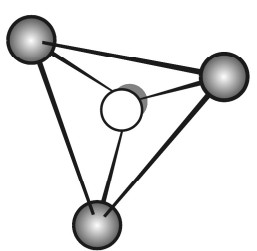

Se1

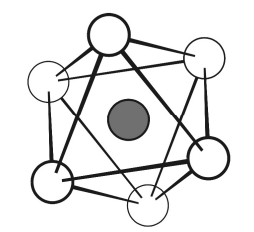

$\mathrm{Mg}$

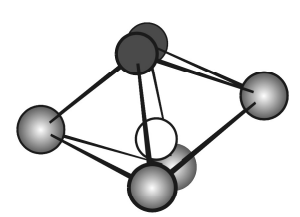

$\mathrm{Se} 2$
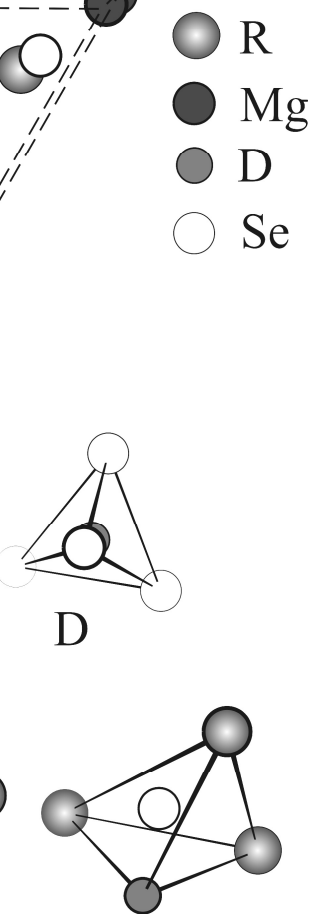

Se3

Fig. 1 Unit cell of the structure of the $R_{3} \mathrm{Mg}_{0.5} D \mathrm{Se}_{7}(R=\mathrm{Ce}, \mathrm{Pr} ; D=\mathrm{Si}, \mathrm{Ge})$ compounds and coordination polyhedra of the $R, \mathrm{Mg}, D, \mathrm{Se} 1, \mathrm{Se} 2$, and $\mathrm{Se} 3$ atoms. 


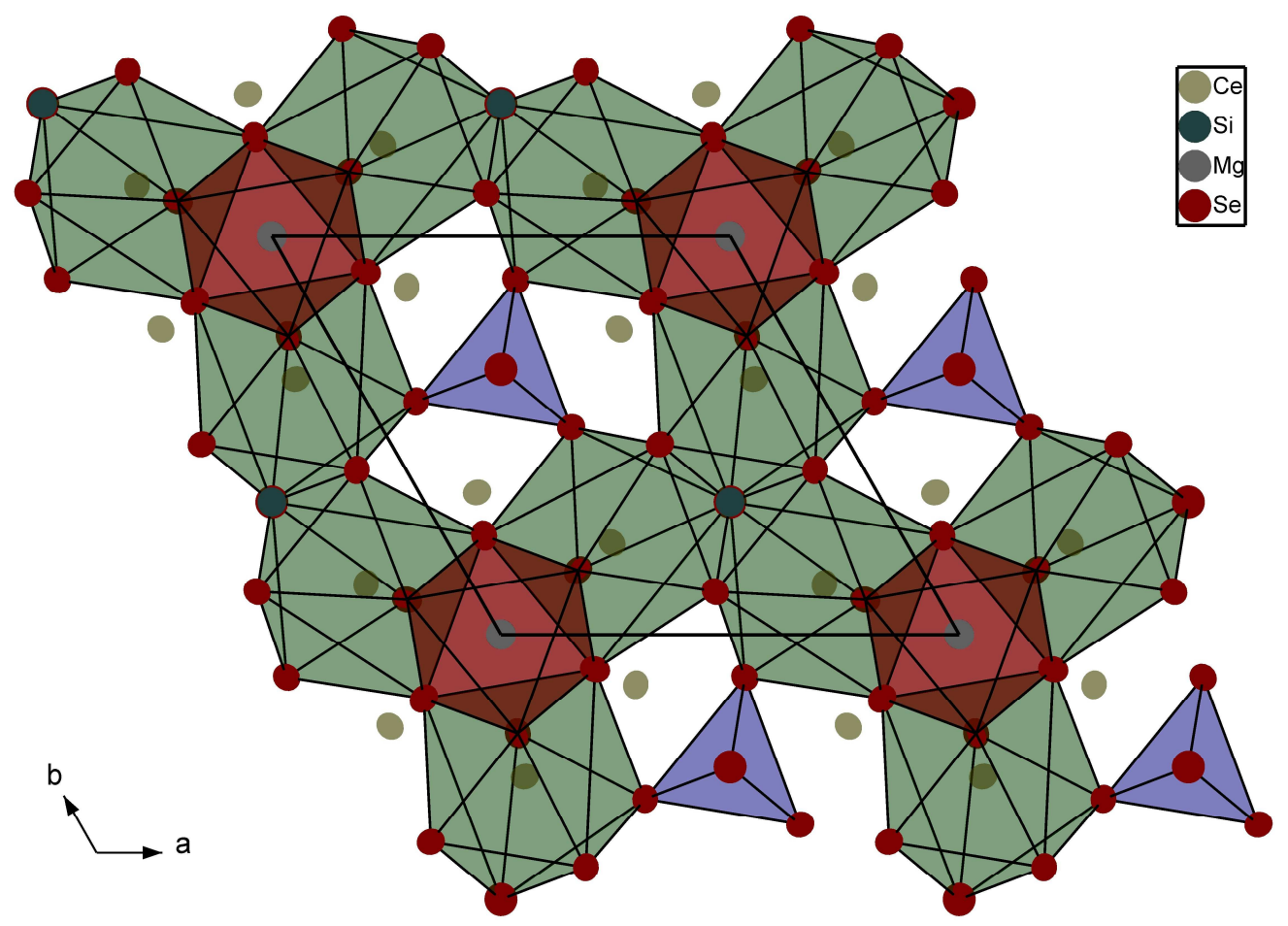

Fig. 2 Packing of $\left[\mathrm{CeSe} 1_{1} \mathrm{Se} 2_{4} \mathrm{Se} 3_{3}\right]$ bi-capped trigonal prisms, [MgSe $\left.2_{6}\right]$ octahedra and $\left[\mathrm{SiSe} 1_{1} \mathrm{Se} 3_{3}\right]$ tetrahedra in the structure of $\mathrm{Ce}_{3} \mathrm{Mg}_{0.5} \mathrm{SiSe}_{7}$.

The crystal structures of $R_{3} \mathrm{Mg}_{0.5} D \mathrm{Se}_{7}(R=\mathrm{Ce}, \mathrm{Pr}$; $D=\mathrm{Si}, \mathrm{Ge})$ may be seen as built up by different coordination polyhedra, e.g. $\left[R \mathrm{Se} 1_{1} \mathrm{Se} 2{ }_{4} \mathrm{Se} 3_{3}\right]$ bi-capped trigonal prisms, [MgSe $\left.2_{6}\right]$ octahedra and [DSe $\left.1_{1} \mathrm{Se} 3_{3}\right]$ tetrahedra (see Fig. 2), sharing common corners or faces. Structurally the $R_{3} \mathrm{Mg}_{0.5} D \mathrm{Se}_{7}$ ( $R=\mathrm{Ce}, \mathrm{Pr} ; D=\mathrm{Si}, \mathrm{Ge}$ ) compounds investigated in the present paper are members of a large family of compounds with the general formula $R_{3} M D X_{7}$ (space group $P 6_{3}$ ), where $R=$ lanthanide element; $M=1$ mono-valent element (for example, $\mathrm{Cu}$ or $\mathrm{Ag}$ ), 1/2 of a di-valent element (for example, $\mathrm{Mg}, \mathrm{Fe}$ or $\mathrm{Mn}$ ), or $1 / 4$ of a four-valent element ( $\mathrm{Si}, \mathrm{Ge}, \mathrm{Sn}) ; D=\mathrm{Si}, \mathrm{Ge}$, $\mathrm{Sn}$; and $X=\mathrm{S}$, Se. All these compounds have similar structural motifs. The packing of $\left[R X_{8}\right]$ trigonal prisms and $\left[D X_{4}\right]$ tetrahedra is similar for all the structures and the differences are only in the occupation of the octahedra located along the $c$ axis. The compounds can be divided into three groups (see [1]). The $R_{3} \mathrm{Mg}_{0.5} D \mathrm{Se}_{7}(R=\mathrm{Ce}, \mathrm{Pr} ; D=\mathrm{Si}, \mathrm{Ge})$ compounds belong to the third group. Two series of the recently investigated $R_{3} \mathrm{Mn}_{0.5} \mathrm{GeS}_{7}(R=\mathrm{Y}, \mathrm{Ce}, \mathrm{Pr}, \mathrm{Nd}, \mathrm{Sm}, \mathrm{Gd}$, $\mathrm{Tb}, \mathrm{Dy}, \mathrm{Ho}$, and Er) [11] and $R_{3} \mathrm{Fe}_{0.5} \mathrm{GeS}_{7}(R=\mathrm{Y}, \mathrm{La}$, Ce, Pr, Sm, Gd, Tb, Dy, Ho, Er, and Tm) [12] compounds are also members of the third group of $R_{3} M D X_{7}$.

\section{References}

[1] L.D. Gulay, M. Daszkiewicz, Ternary and Quaternary Chalcogenides of $\mathrm{Si}, \mathrm{Ge}, \mathrm{Sn}, \mathrm{Pb}$, and In, in: K.A. Gschneidner Jr., J.-C.G. Bünzli, V.K. Pecharsky (Eds.), Handbook on the Physics and Chemistry of Rare Earths, Elsevier B.V., Amsterdam, 2011, Vol. 41, Ch. 250, pp. 157-273.

[2] K. Mitchell, J.A. Ibers, Chem. Rev. 102 (2002) 1929-1952.

[3] A. Michelet, J. Flahaut, C. R. Seances Acad. Sci. C 269 (1969) 1203-1205.

[4] R.L. Gitzendanner, C.M. Spencer, F.J. DiSalvo, M.A. Pell, J.A. Ibers, J. Solid State Chem. 131 (1997) 399-404.

[5] M.R. Huch, L.D. Gulay, I.D. Olekseyuk, J. Alloys Compd. 424 (2006) 114-118.

[6] CrysAlis CCD and CrysAlis RED, version 1.171.30.3, Oxford Diffraction Ltd., Abingdon, Oxfordshire, United Kingdom, 2006.

[7] G.M. Sheldrick, SHELXS97 and SHELXL97, Programs for the Solution and the Refinement of Crystal Structures, University of Göttingen, Germany, 1997. 
[8] A.L. Spek, PLATON, A Multipurpose Crystallographic Tool, Utrecht University, Utrecht, The Netherlands, 2007.

[9] G. Collin, P. Laruelle, C. R. Seances Acad. Sci. C 270 (1970) 410-412.

[10] N. Wiberg, Lehrbuch der anorganischen Chemie, Walter de Gruyter, Berlin, 1995, pp. $1838-1841$.
[11] M. Daszkiewicz, O.V. Marchuk, L.D. Gulay, D. Kaczorowski, J. Alloys Compd. 610 (2014) 258-263.

[12] M. Daszkiewicz, Yu.O. Pashynska, O.V. Marchuk, L.D. Gulay, D. Kaczorowski, J. Alloys Compd. 616 (2014) 243-249. 